\title{
Remote Measurement and Monitoring of Critical Washing Process Data directly inside the Washing machine drum
}

\author{
M. Lazzaroni(*), E. Pezzotta (*), G. Menduni(**), D. Bocchiola(**), D. Ward (***) \\ $\left({ }^{*}\right)$ Politecnico di Milano - Dipartimento di Elettrotecnica, Piazza Leonardo Da Vinci, 32 - 20133 Milano - Italy. \\ (**) Politecnico di Milano - DIIAR, Pjazza Leonardo Da Vinci, 32 - 20133 Milano - Italy. \\ (***) The Open University, Faculty of Technology, Walton Hall, Milton Keynes, England
}

\begin{abstract}
The system proposed allows the remote sensing of pressure inside the wash load of a domestic washing machine via a wireless data acquisition system.

The system is based on a piezoresistive pressure transducer and a matched pair of battery operated hybrid transmitters and receivers with a working frequency of 433MHz. The transducer and transmitter are housed within a small plastic sphere designed to follow the wash load during its movement in the washing machine drum. A small block probe is fitted to the surface of the sphere to pick up the surface dynamic pressure of the sphere and relate this to the local surface velocity. The receiver is mounted on the outside of the washing machine where it picks up the modulated signal from the transmitter and stores it through a dedicated data acquisition system.

The system has been designed to offer the maximum flexibility and is therefore applicable to many other remote sensing circumstances including those outside domestic appliances.
\end{abstract}

\section{INTRODUCTION}

The removal of soil in the textiles industry is of prime importance for the washing appliance consumer and for the manufacturer the world-wide domestic washing appliance business alone is worth billions of US\$.

However, there is a fundamental gap in knowledge relating soil removal to the fluid dynamics within the washing machine and especially near the surface of the soiled textile. In fact flow velocity measurements carried out within the wash load have so far have been achieved by a combination of permeability measurements of the textile

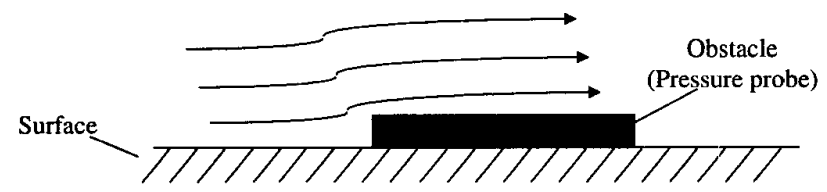

Fig. 1 - Small block probe to measure local flow velocity near the wall. fabric in static conditions and using reactor modeling in dynamic conditions [1]. The main drawback is that these methods are bulk rather than local measurements.

However, recent research indicates $[2,3]$ that flow on the outside of the wash load could also be important and flow velocities near the weave surface could therefore be much higher.

Thus the knowledge of the hydrodynamic conditions, especially the surface or wall velocity, becomes decidedly more interesting. A system [4] has therefore been developed which measures the dynamic pressure within the washing drum during wash load motion and attempts to extrapolate information about the local water velocity.

\section{MEASUREMENT METHOD}

The measurement method is based on a small block probe mounted on the surface of a sphere that obstructs local flow velocity and therefore imparts some of this lost energy on the surface of the probe $[5,6]$. This method is depicted in Fig 1.

The probe [7] consists of two thin $(0.1-0.15 \mathrm{~mm})$ brass sheets bonded together with special twin sided adhesive tape (type 3M VHB9460, thick $=0.05 \mathrm{~mm}$ ) and both suitably shaped to provide dynamic (and static) pressure tappings where the fluid is trapped. The lower half of the probe is bonded to the sphere wall and is shaped so as to facilitate the capture of local wall flow. On top of this side of the probe is bonded the upper half of the probe, which creates the 'mouth'. A cross section of the assembled probe is given below in Fig. 2a.

The probe is triangular shaped [8] and each side has a dynamic pressure tapping that is connected to the pressure transducer housed in the sphere. At the centre of the probe is a fourth tapping, which can be used to monitor the local static pressure (figure $2 b$ ).

Pictures of the probe are shown in Fig. 3.

A series of small needles are installed at the rear of the probes i.e. inside the sphere that transfer the pressure, via small silicone tubing, to the pressure transducer as shown in Fig. 4.

\section{0-7803-5890-2/00/\$10.00 (c) 2000 IEEE}




\section{DESCRIPTION AND OPERATION OF THE SYSTEM}

The system is divided into two parts: part 1 consists of a hollow plastic sphere which houses the needles, silicone tubing, pressure transducer, battery and transmitter with integrated antenna while part 2 is external to the drum and consists of the receiver section of the system. The sphere, shown below open, has the transmitter and transducer fitted in one half and the other half occupied by the battery and external connections. The size of the sphere is approximately $95 \mathrm{~mm}$ in diameter and weighs less than $200 \mathrm{~g}$ fully assembled. Fig. 5 depict the two parts of the sphere.

The receiver section includes the hybrid receiver (Aurel mod. RF290A-300), relative power supply, a sample and hold board (mod. SC2040), a PCMCII data acquisition card (mod. DAQcard-A-16E-4 E), both by National Instruments, and the data acquisition program developed using National Instruments LABVIEW release 5.1. The data acquisition card and SW being installed on a IBM 600 Thinkpad laptop where the experimental data was both acquired and processed.

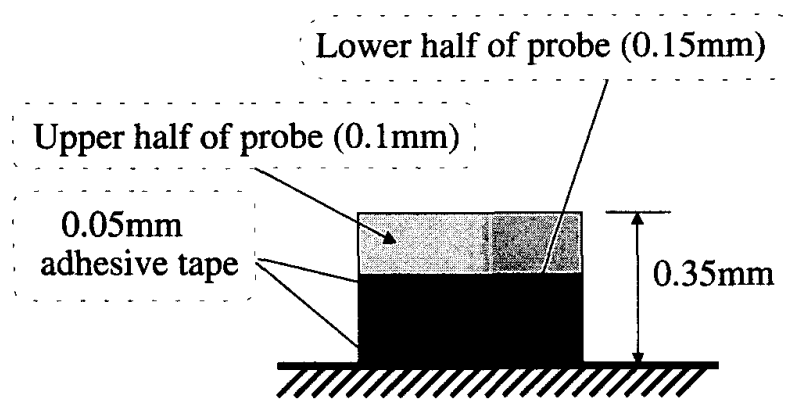

a)

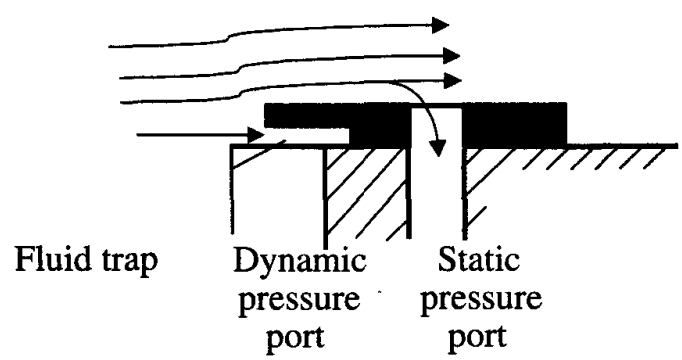

b)

Fig. 2 - Cross-section of Small Block Probe.
The pressure transducer detects the dynamic pressure on the small block probe as if it were a small flat Pitot tube [9] and converts it into a voltage signal, after which it is sent to a voltage to frequency converter. The output frequency is then modulated and transmitted outside the washing drum via a miniature battery operated radio transmitter (Aurel mod. TX-SAW - I.A.) at $433 \mathrm{MHz}$ (Fig. 6-a).

The matched radio receiver (Fig. 6-b) is located within a short distance $(<5 \mathrm{~m})$ on the outside of the washing machine and its output signal is sent to the sample and hold board and then to the data acquisition card and laptop where the experimental data is stored and processed.

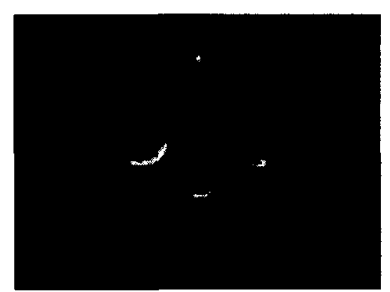

Lower Half of Probe

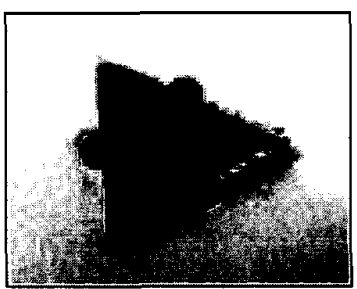

Assembled Probe

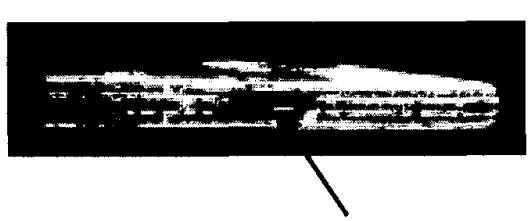

Capture or probe "mouth"

Fig. 3 - Assembled probe.

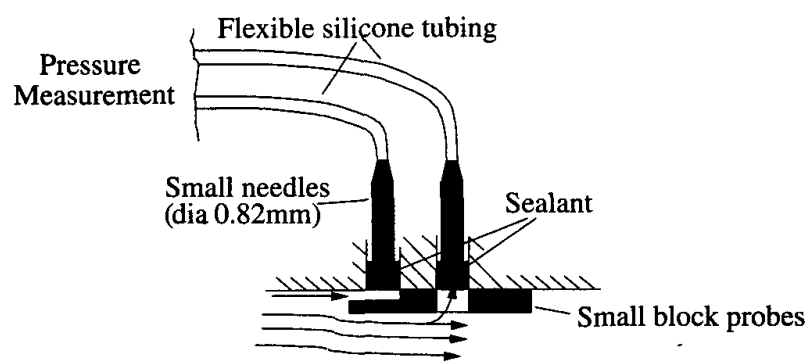

Fig. 4 - Probe connection to Pressure Transducer 
To start the acquisition the sphere is placed in the washing drum with the wash load and the data acquisition started. The washing cycle is activated and the data supplied by the pressure transducer transferred to the laptop mass memory where it is subsequently retrieved and

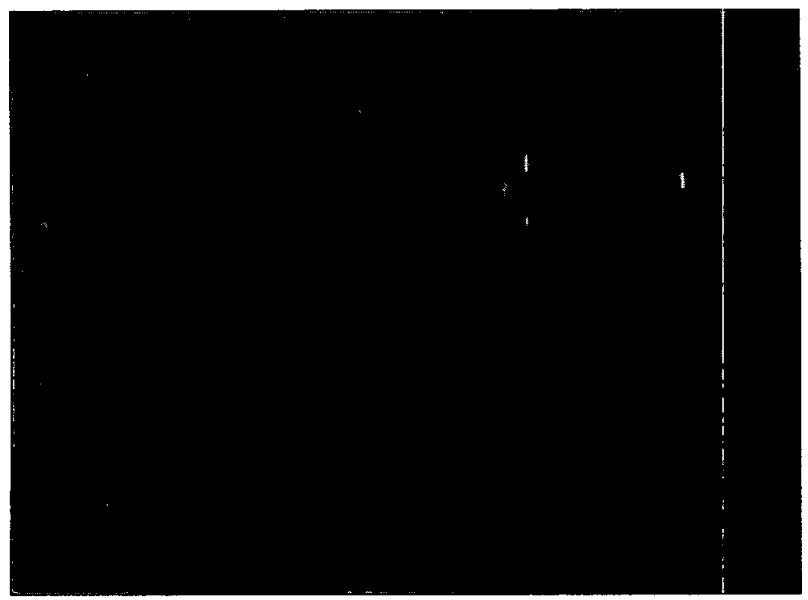

Fig. 5 - Inside the sphere showing battery, transducer, transmitter and $\mathrm{V}-\mathrm{F}$ converter.

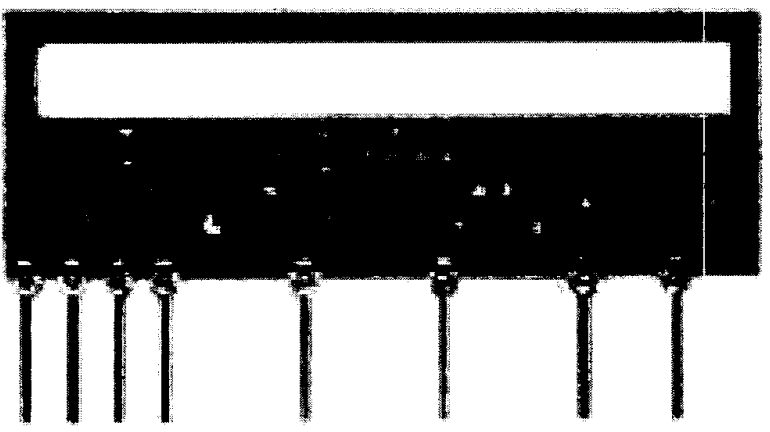

a)

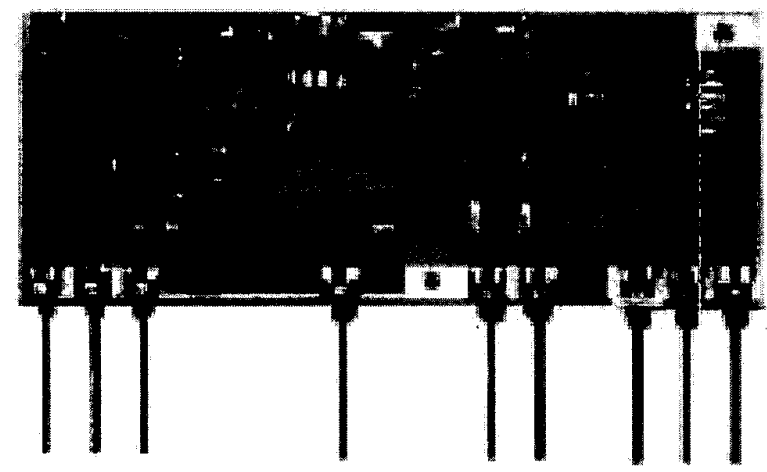

b)

Fig. 6-a) Transmitter circuit, b) Receiver circuit. processed. The complete system is shown in Fig. 7.

\section{CALIBRATION}

Before experimentation two calibration or characterization procedures were involved, these being:

- Characterisation of the sphere;

- Electrical characterisation of the transmitter-receiver and data acquisition section.

In the first case the sphere was calibrated by subjecting the probe to a series of specific flow conditions [10] and using a Yokogawa MT120 digital pressure manometer to record the dynamic pressure. In this way it was possible to relate flow rate, local flow velocity and dynamic pressure. Typical calibration conditions were $\mathrm{Q}=2-10 \mathrm{E}-3 \mathrm{~s}^{-1}, \mathrm{Pd}=100$ $500 \mathrm{~Pa}, \mathrm{u}=0.2-1 \mathrm{~ms}^{-1}$. The test ring is shown schematically in Fig. 8 .

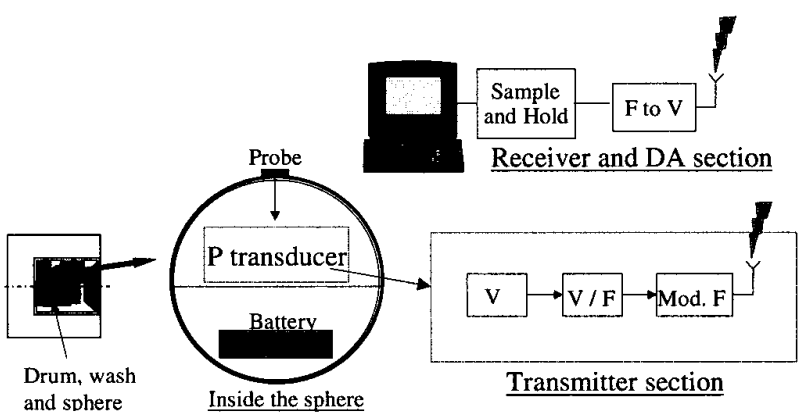

Fig. 7 - The Realized System.

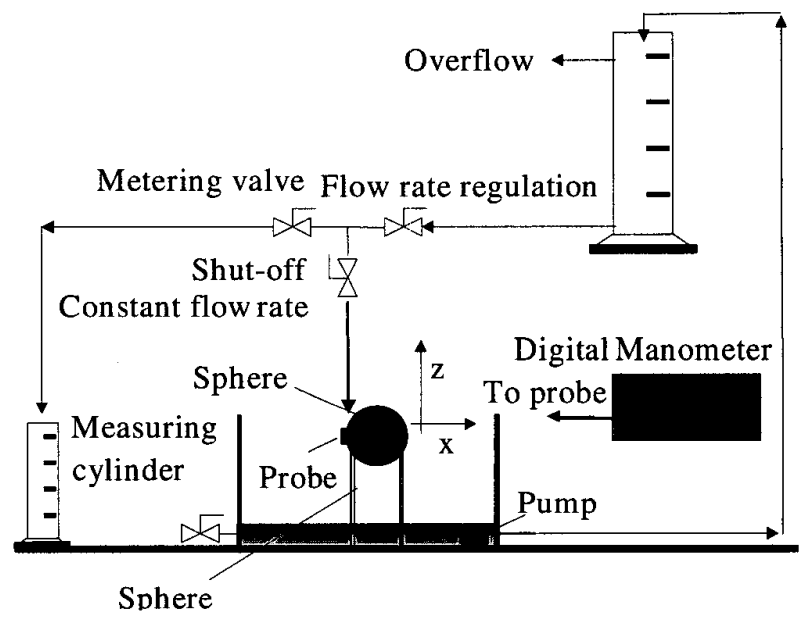

Fig. 8 - Calibration of the Probe and Transducer. 


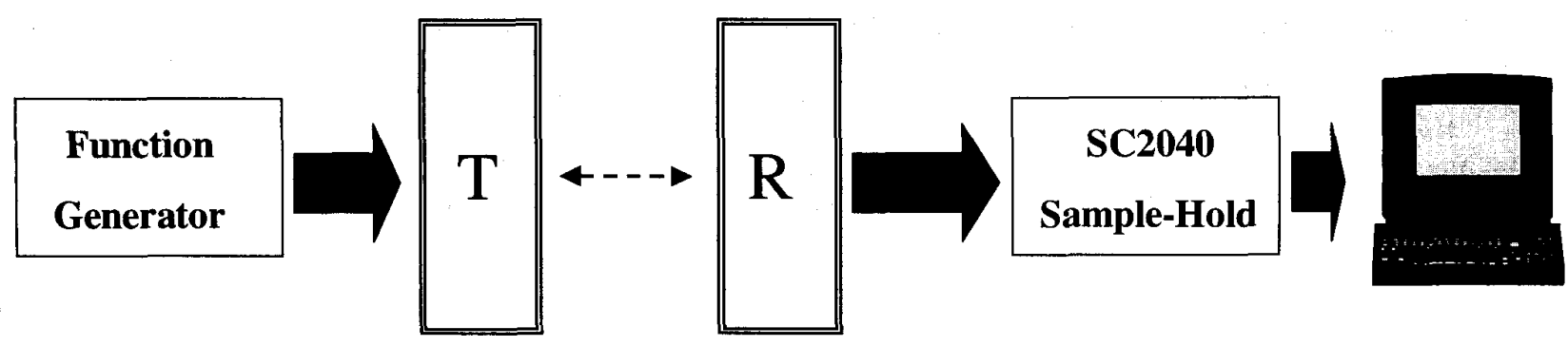

Fig. 9 - Calibration of the transmitter-receiver section.

Once the probe was characterised the transmitter and receiver connection was calibrated by supplying several different waveforms to the transmitter input (i.e. after the $\mathrm{V}-\mathrm{F}$ converter) and recording the received signal. This test was repeated for a series of signal conditions in order to be able to fully characterise the system and quantify parameters such as the gain, noise, signal-to-noise ratio etc.

The test set-up is illustrated in the Fig. 9 followed by a series of traces obtained during the procedure depicted in figures 10,11 and 12 .

The gain of the system was found to be sufficiently flat.

In Fig. 13 is shown the experimental transfer function of the system. The band pass is sufficient to handle the signal present during the real experimental activity.

Fig. 14 depict a typical acquisition performed by the system during an experimental set up in real condition.

The acquisition was obtained by a sampling rate of $6 \mathrm{KSa} / \mathrm{s}$ and 15 second long (only the first second of phenomena is displayed in the figure).

\section{CONCLUSIONS}

The system is capable of measuring pressure within the washing machine and providing an estimation of the flow conditions within the drum during the rotation of the wash load. Perhaps the most appealing part of this work is that the system certainly lends it self to a whole series of transducer and monitoring applications within the washing machine e.g. temperature, humidity etc.

Also the cost of the whole system is not prohibitive since it is based on off-the-shelf industrial solutions thus providing both reliability and cost effectiveness.

Clearly the advent of smaller transducers and hybrid wireless monitoring systems will push this type of solution even further, even to the stage where the garments themselves will have not only identification but also monitoring features.

Further experimental activity is planned in order to validate the project and to verify the utility in obtain new data concerning the washing.

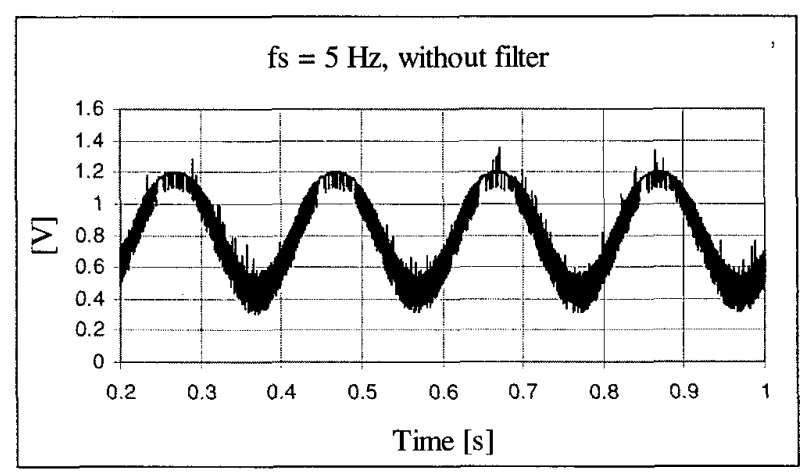

a)

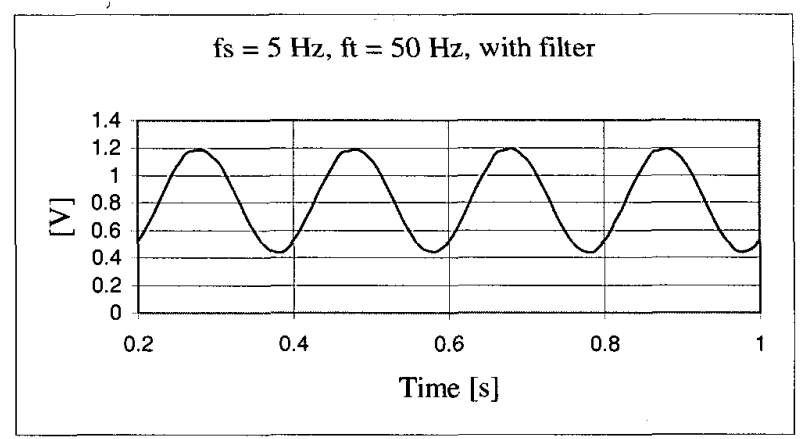

b)

Fig. 10 - Examples of received signal (Sinusoidal input signal).

\section{REFERENCES}

[1] Hydrodynamic and Mass Transfer in Domestic Drumtype Fabric Washing Machines, L.D.M. Van Den Brekel, Delft University of Technology, 1987.

[2] Introductory Evaluation of Soil Removal Conditions, D. Ward, Internal Whirlpool Report, April 1999.

[3] D. Ward, "Modelling of a Horizontal-Axis Domestic 
Washing Machine", Journal of the Textile Institute, Autumn 1999.

[4] D. Ward G. Menduni, M. Viterbo, M. Lazzaroni, "Microelectronic Sensor for the Measurement of Shear Stress within a Domestic Washing Machine Drum", Giornate IMAPS-Italy 1999, Milano, 5-6 Luglio 1999, Politecnico di Milano - Campus Bovisa.

[5] Gaudet et al. "Calibration and Use of a Triangular Yawmeter for Surface Shear Stress and Flow Direction Measurement", Proc. $2^{\text {nd }}$ Intern. Conf. On Experirnental Fluid Mechanics, Torino, 1994.

[6] K. G. Winter, "An Outline of Techniques Available for the Measurement of Skin Friction in Turbulent Boundary Layers", Royal Aircraft Est., Prog. Aerospace Sci. Vol. 18, pp. 1-57, Pergamon Press, 1977.

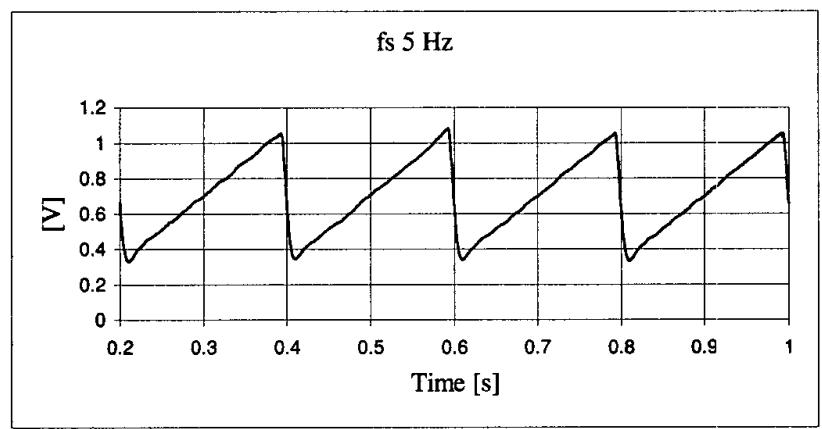

Fig. 11 - Examples of received signal (Ramp like input signal).

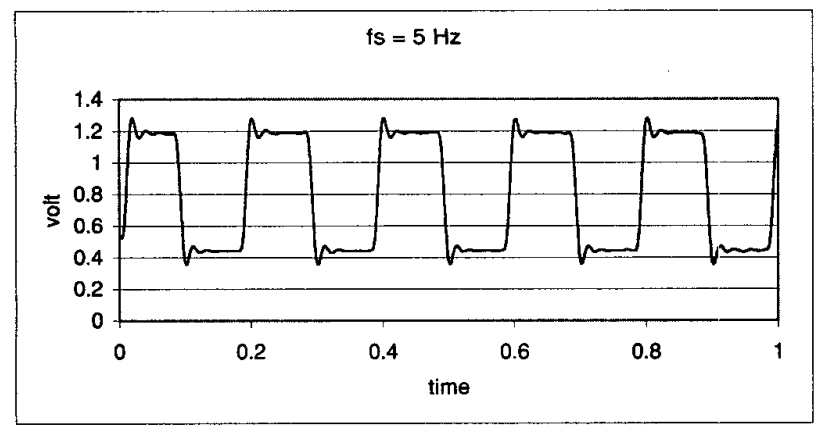

Fig. 12 - Examples of received signal (Square input signal).
[9] The determination of Turbolent Skin Friction by Means of Pitot Tubes, J. R. Aeronaut Soc., Vol. 58, pp. 109$121,1953$.

[10] Fluid Mechanics Measurements, Taylor and Francis, $2^{\text {nd }}$ edition, 1998.

[7] D. Bocchiola, "Measurement of Wall Skin Friction in Cylindrical Ducts", Engineering degree thesis $\mathrm{N}^{\circ}$ 611090, Politecnico di Milano, 1998.

[8] M. Viterbo, "Boundary layer probes used for the measurement of local wall shear stress - A theoretical and experimental Analysis", Engineering degree thesis, Mat. N 599289, Politecnico di Milano, 1999.

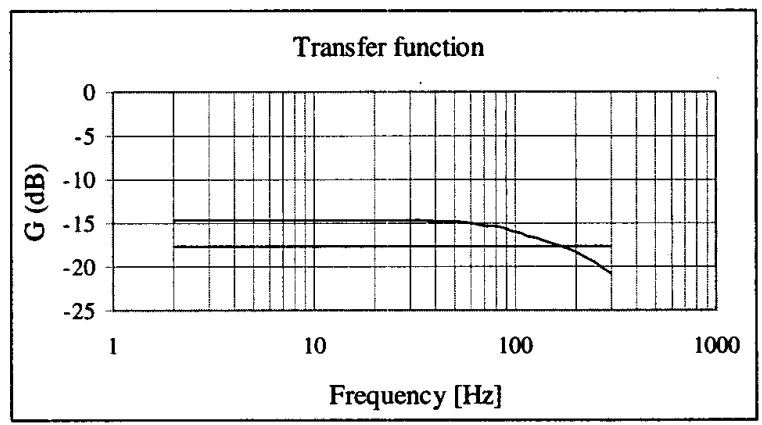

Fig. 13 - Transfer function of the realized system.

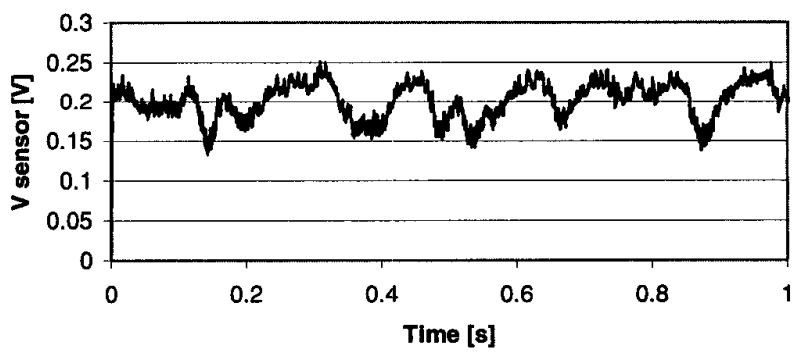

Fig. 14 - Examples of Received signals from the pressure sensor. 